\title{
Catatonic features in children and adolescents with $N$-methyl-D-aspartate receptor antibody encephalitis
}

\author{
Michael Eyre, Anya Kaushik, Elizabeth Barrett, Mary D. King, Thomas Pollak, Russell C. Dale, Susan Byrne* \\ and Ming Lim*
}

\begin{abstract}
Catatonia is a psychomotor dysregulation syndrome of diverse aetiology, increasingly recognised as a prominent feature of $\mathrm{N}$ methyl-D-aspartate receptor antibody encephalitis (NMDARE) in adults. No study to date has systematically assessed the prevalence and symptomatology of catatonia in children with NMDARE. We analysed 57 paediatric patients with NMDARE from the literature using the Bush-Francis Catatonia Rating Scale. Catatonia was common (occurring in $86 \%$ of patients), manifesting as complex clusters of positive and negative features within individual patients. It was both underrecognised and undertreated. Immunotherapy was the only effective intervention, highlighting the importance of prompt recognition and treatment of the underlying cause of catatonia.
\end{abstract}

\section{Keywords}

Clinical neurology; inpatient treatment; neuroimmunology; organic syndromes; drug interactions and side effects.

\section{Copyright and usage}

(C) The Author(s), 2020. Published by Cambridge University Press on behalf of The Royal college of Psychiatrists. This is an Open Access article, distributed under the terms of the Creative Commons Attribution-NonCommercial-ShareAlike licence (http://creativecommons.org/licenses/by-nc-sa/4.0/), which permits non-commercial re-use, distribution, and reproduction in any medium, provided the same Creative commons licence is included and the original work is properly cited. The written permission of Cambridge University Press must be obtained for commercial re-use.
Psychiatrists are increasingly involved in the diagnosis and management of patients with autoimmune encephalitis, and many patients present initially to psychiatrists. The commonest cause of autoimmune encephalitis in children and young people is $N$-methyl-Daspartate receptor (NMDAR) antibody encephalitis (NMDARE), in which antibodies against the GluN1 subunit of NMDAR cause a severe and progressive neuropsychiatric syndrome characterised in the later stages by encephalopathy, seizures and movement disorder, but frequently presenting in the early stages with prominent psychiatric or behavioural symptoms that can be difficult to differentiate from primary psychiatric disease. ${ }^{1}$

The psychopathological features of NMDARE are well characterised in adults, ${ }^{2}$ but less understood in children and adolescents, despite over a third of cases occurring in this group. ${ }^{3}$ Diagnosis of autoimmune encephalitis in patients presenting with psychiatric symptoms is often delayed; ${ }^{4}$ as earlier treatment is associated with a better outcome in both children and adults ${ }^{3,5}$ recognition of psychiatric features in children is clearly important. Catatonia is frequently reported in adults with NMDARE but has never been systematically evaluated in children with the disease. ${ }^{4,6,7}$ We therefore analysed individually reported paediatric patients with NMDARE to assess the prevalence and symptomatology of catatonia and the relationship to clinical characteristics and outcome.

\section{Method}

We previously reported a MEDLINE literature search for firstepisode cases of paediatric NMDARE, identifying individually reported data in 80 children ( $\leq 17$ years) across 34 publications (see Byrne et al Appendix e-1). ${ }^{5}$ Only cases of patients with adequate detailed individualised information to reveal specific clinical characteristics and outcome were included. Outcome was dichotomised into either complete recovery (defined as modified Rankin Scale score of zero) or incomplete recovery at final follow-up, assessed

* Joint last authors in each patient by three independent reviewers (S.B., M.L. and R.C.D.). In the present study we conducted a secondary analysis of this cohort to extract catatonic features using the validated screening instrument of the Bush-Francis Catatonia Rating Scale (BFCRS), comprising a checklist of 14 features in which the presence of $\geq 2$ suggests a diagnosis of catatonia. ${ }^{8}$ The BFCRS was applied to reports containing adequate detailed descriptions of movement and behaviour by two independent reviewers (S.B. and A.K.). The Mann-Whitney $U$-test was used to analyse group differences in BFCRS total score according to dichotomised clinical characteristics. Multivariate logistic regression was used to determine the independent association of BFCRS total score with complete recovery.

\section{Results}

In total, the cases of 57 patients were analysed (median age 8 years, range 1.3-17; 40 females). A median of three catatonic features were identified per patient (range 0.5-10). Figure 1 details the frequency of each feature within the cohort, and co-occurrences of feature pairs within individual patients (shown as connections between features). Two or more catatonic features were present in 49/57 patients (86\%), of whom 34 (69\%) had both positive and negative features, 10 (20\%) had positive features only, and $5(10 \%)$ had negative features only.

Catatonia was recognised by the reporting physician in only $16 /$ 49 patients (33\%). Patients with recognised catatonia were older (median $14.5 v .7$ years, $P<0.001$ ) and had a greater number of positive (median $2 v .1, P=0.034)$ and negative $(3 v .1 .5, P<0.001)$ catatonic features. Five $(31 \%)$ were treated with lorazepam, without sustained response. Eleven (69\%) were treated with antipsychotic medications, with adverse effects reported in five (45\%): central nervous system depression and extrapyramidal signs with haloperidol $(n=3)$, worsening dystonia with olanzapine $(n=1)$, and orofacial dyskinesia with risperidone $(n=1)$. In all patients, symptoms of catatonia improved only after initiation of immunotherapy.

Associations of clinical characteristics with catatonia score are detailed in Table 1. Higher catatonia score was associated with a 


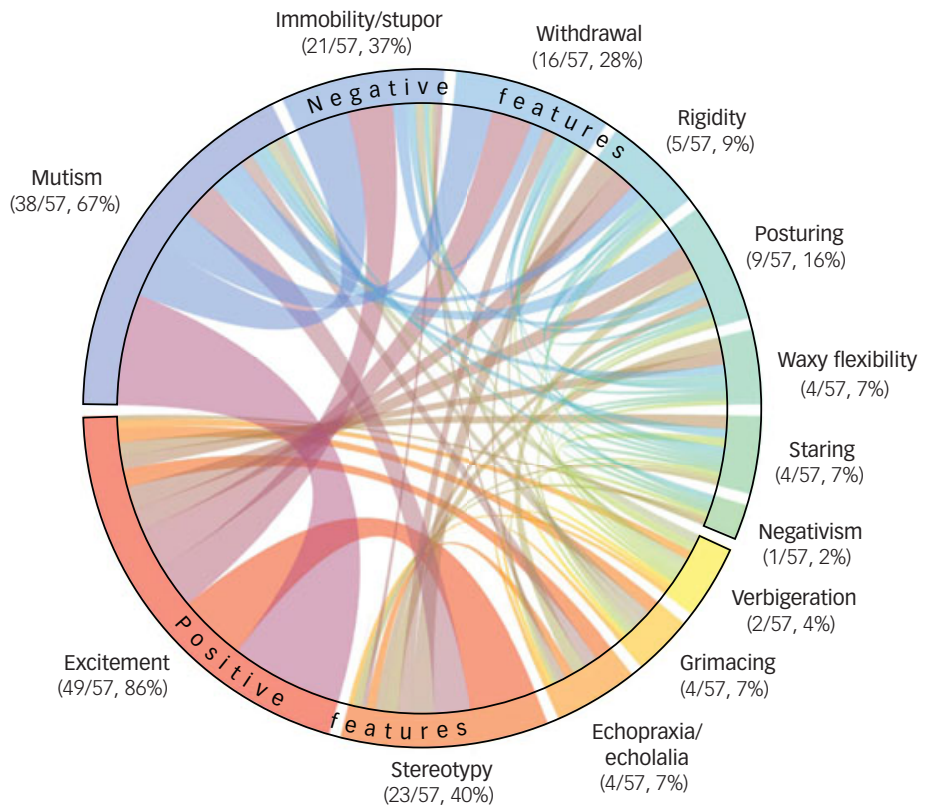

Fig. 1 Catatonic features and clinical characteristics in children and adolescents with $N$-methyl-D-aspartate receptor antibody encephalitis. Frequency of catatonic features and co-occurrence of features within individual patients.

Chord diagram: blue-green node segments represent negative features and red-yellow segments represent positive features of catatonia. $n / N(\%)$ indicates the frequency of feature occurrence within the whole cohort. Arc connections represent flow between features, such that arc thickness is proportional to the number of individual patients in which connected features co-occurred. Mannerisms were also reported in one patient, excluded from figure because of the absence of co-occurrence with any other features. Figure produced in Python 3.6 with code adapted from Matplotlib-chord-diagram (Feng Wang, MIT).

clinical history of prodromal infectious illness (median score $4 \mathrm{v} .3$, $P=0.03)$ and complete recovery from NMDARE ( $4 v .3, P=0.009)$. Complete recovery occurred in $25 / 57$ patients (44\%). There was no significant difference in follow-up duration between those with complete recovery (median 7 months, interquartile range $(\mathrm{IQR})=$ 4.5-17, range 2-54) and those without (median 9 months, IQR $=3-18.75$, range $1.3-33)(P=0.764)$. To evaluate the independent association of catatonia score with complete recovery (controlling for differences in age and other clinical characteristics) we fitted a multivariate logistic regression model (Supplementary Table 1 available at https://doi.org/10.1192/bjo.2020.55). The presence of each additional catatonic feature $(+1$ BFCRS score) was associated with 2.08 (CI 1.20-3.60) times increased odds of complete recovery $(P=0.009)$.

\section{Discussion}

In total, $86 \%$ of children and adolescents with NMDARE had signs and symptoms consistent with catatonia, in keeping with previous reports of catatonia in $19-88 \%$ of NMDARE patients. ${ }^{4,6,7}$ The symptomatology was complex, with $69 \%$ of affected patients manifesting both positive (hyperkinetic) and negative (hypokinetic) features. Only $33 \%$ were recognised as catatonic by the reporting physician. These were predominantly neurologists, who may tend to interpret movement disorders within a purely neurological paradigm, failing to recognise catatonia as a neuropsychiatric syndrome bridging across traditional symptom domains. Catatonia is not an aetiological diagnosis, but rather a psychomotor dysregulation syndrome with many causes; determination of the cause in the individual patient is of key clinical importance.

Symptomatic treatment with lorazepam was ineffective in this cohort, in keeping with previous reports in adult NMDARE, ${ }^{6}$ and in contrast to the majority of patients presenting to general psychiatry with catatonia, $88 \%$ of whom improve with lorazepam. ${ }^{9}$
Lorazepam acts by potentiating the effect of the inhibitory neurotransmitter $\gamma$-amino-butyric acid (GABA) at the GABA-A receptor $^{10}$ the hypothesised inactivation of GABAergic neurons in $\mathrm{NMDARE}^{11}$ may explain the reduced efficacy of the drug in this disease. Failure to respond to lorazepam should therefore prompt careful evaluation for an underlying neurobiological disorder in patients who are catatonic. Adverse effects of antipsychotics occurred in $45 \%$ of those treated, in keeping with previous reports in paediatric NMDARE. ${ }^{12}$ The reasons for this remain unclear, but likely result from complex interactions of cortical and subcortical NMDAR hypofunction with dopaminergic and other neurotransmitter pathways, confounded by a host of other factors experienced by the critically ill patient. The only effective intervention for catatonia in this cohort was early initiation of immunotherapy.

In our exploratory analysis of clinical characteristics associated with catatonia, contrary to previous reports of worse outcome in paediatric NMDARE with catatonia, ${ }^{13}$ we surprisingly found complete recovery was associated with a greater number of catatonic features during the illness course. This is consistent with findings in adults with NMDARE, in which both status epilepticus and death occurred more often in patients without catatonia. ${ }^{6}$ Recent research implicates dysregulation and hyperactivity of the cortical supplementary and presupplementary motor areas as a pathophysiological mechanism in catatonia; ${ }^{9}$ it may be that patients who are more severely encephalopathic (who tend to have worse outcome) are unable to support any organised output of cortical motor circuits, and so are unable to manifest the behavioural signs of catatonia.

This retrospective study has a number of limitations, including scarcity of detailed information available in case reports for catatonia scoring, bias towards atypical cases in such reports, variable terminology used by authors (typically non-psychiatrists) to describe psychomotor signs and symptoms, and diagnosis that was not always confirmed with cerebrospinal fluid testing. In addition, our analysis of associations with clinical features was exploratory and uncontrolled for multiple comparisons. In summary, we found 
Table 1 Associations of clinical characteristics with catatonia score (Mann-Whitney $U$-test) ${ }^{\mathrm{a}}$

Bush-Francis Catatonia Rating $n / N(\%) \quad$ Scale total score, median (range)

$P$

\begin{tabular}{|c|c|c|}
\hline \multicolumn{3}{|l|}{ Gender } \\
\hline Female & $38 / 57$ (67) & $3(0.5-10)$ \\
\hline Male & 19/57 (33) & $3(1-9.5)$ \\
\hline \multicolumn{3}{|l|}{ Tumour } \\
\hline Present & $5 / 56(8.9)$ & $3(2-10)$ \\
\hline Absent & $51 / 56$ (91) & $3(0.5-9.5)$ \\
\hline \multicolumn{3}{|l|}{$\begin{array}{l}\text { Infectious } \\
\text { prodrome }\end{array}$} \\
\hline Present & 19/57 (33) & $4(1-10)$ \\
\hline Absent & $38 / 57$ (67) & $3(0.5-9)$ \\
\hline \multicolumn{3}{|l|}{$\begin{array}{l}\text { Psychotic } \\
\text { features }^{\text {b }}\end{array}$} \\
\hline Present & 17/57 (30) & $4(1-9)$ \\
\hline Absent & 40/57 (70) & $3(0.5-10)$ \\
\hline \multicolumn{3}{|l|}{$\begin{array}{l}\text { Movement } \\
\text { disorder }^{\mathrm{C}}\end{array}$} \\
\hline Present & 17/57 (30) & $3(2-10)$ \\
\hline Absent & 40/57 (70) & $3(0.5-9.5)$ \\
\hline \multicolumn{3}{|l|}{ Seizures ${ }^{c}$} \\
\hline Present & 18/57 (32) & $3(1-10)$ \\
\hline Absent & 39/57 (68) & $3(0.5-9)$ \\
\hline \multicolumn{3}{|l|}{ MRI brain } \\
\hline Normal & 18/33 (55) & $4(2-10)$ \\
\hline Abnormal & $15 / 33(45)$ & $3(1-6.5)$ \\
\hline \multicolumn{3}{|c|}{ pleocytosis $^{d}$} \\
\hline Present & 22/34 (65) & $3.25(1-10)$ \\
\hline Absent & $12 / 34(35)$ & $3(2-5.5)$ \\
\hline \multicolumn{3}{|l|}{ Recovery } \\
\hline Complete & 25/57 (44) & $4(1.5-10)$ \\
\hline Incomplete & 32/57 (56) & $3(0.5-6.5)$ \\
\hline
\end{tabular}

$\mathrm{MRI}$, magnetic resonance imaging

a. Electroencephalogram was abnormal in all 34 patients with data reported, and therefore not included in the analysis.

b. Delusions, paranoia, hallucinations or thought disorder reported at any time. c. At presentation to hospital.

d. $>5$ leucocytes $/ \mu$ L in cerebrospinal fluid (CSF).

features consistent with catatonia were highly prevalent in paediatric NMDARE. Catatonia was characterised by mixed or fluctuating symptomatology, resistance to lorazepam and antipsychotic intolerance. Symptoms resolved only with immunotherapy in all cases, highlighting the importance of prompt recognition and treatment of the underlying cause of catatonia.

Michael Eyre (D), Department of Neurology, Great Ormond Street Hospital for Children, UK; Anya Kaushik, Department of Child and Adolescent Psychiatry, South London and Maudsley NHS Foundation Trust, UK; Elizabeth Barrett, St Frances Clinic, Temple Street Children's University Hospital, Ireland; and Department of Child and Adolescent Psychiatry, School of Medicine and Medical Science, University College Dublin, Ireland; Mary D. King, Department of Pediatric Neurology, Temple Street Children's University Hospital, Ireland; and Academic Centre on Rare Disease, School of Medicine and Medical Science, University College Dublin, Ireland; Thomas Pollak, Department of Psychosis Studies, Institute of Psychiatry, Psychology and Neuroscience, King's College London, UK ; Russell C. Dale, Neuroimmunology Group, Kids Neuroscience Centre, Children's Hospital at Westmead, University of Sydney, Australia; Susan Byrne, Children's Neurosciences, Evelina London Children's Hospital, UK; and FutureNeuro at Royal College of Surgeons, Ireland; Ming Lim, Children's Neurosciences, Evelina London Children's Hospital, UK; and Department of Women and Children's Health, School of Life course Sciences, Faculty of Life Sciences and Medicine, King's College London, UK

Correspondence: Ming Lim. Email: ming.lim@gstt.nhs.uk

First received 24 Mar 2020, final revision 9 Jun 2020, accepted 9 Jun 2020

\section{Supplementary material}

Supplementary material is available online at http://doi.org/10.1192/bjo.2020.55

\section{Data availability}

The search criteria and papers included in our previously reported systematic literature review are provided in Appendix e-1 of Byrne et al. ${ }^{5}$

\section{Acknowledgements}

Thanks to Eleni Miltsiou, Victoria Chapman, Helen Barry and Peter Hindley for contributing to ideas highlighted in this study.

\section{Author contributions}

M.E. contributed to data analysis and wrote the first draft of the manuscript. A.K. contributed to study formulation/design and data collection. E.B. and M.D.K. contributed to study formulation/ design. T.P. contributed to data analysis. R.C.D. contributed to data collection and data analysis. S.B. and M.L. contributed to study formulation/design, data collection and data analysis. All authors contributed to data interpretation and drafting, revising and approving the final manuscript.

\section{Declaration of interest}

M.E. reports a travel grant from Terumo BCT, outside the submitted work. R.C.D. reports honoraria from Biogen Idec and Merck Serono as invited speaker, outside the submitted work. M.L. reports personal fees from the Advisory Board Octopharma, and non-financial support from Advisory Board CSL Behring, outside the submitted work. A.K., E.B., M.D.K., T.P. and S.B. report no disclosures.

ICMJE forms are in the supplementary material, available online at https://doi.org/10.1192/bjo. 2020.55

\section{References}

1 Dalmau J, Armangué T, Planagumà J, Radosevic M, Mannara F, Leypoldt A, et al. An update on anti-NMDA receptor encephalitis for neurologists and psychiatrists: mechanisms and models. Lancet Neurol 2019; 18: 1045-57.

2 Al-Diwani A, Handel A, Townsend L, Pollak T, Leite Ml, Harrison PJ, et al. The psychopathology of NMDAR-antibody encephalitis in adults: a systematic review and phenotypic analysis of individual patient data. Lancet Psychiatry 2019; 6: 235-46.

3 Titulaer MJ, McCracken L, Gabilondo I, Armangué T, Glaser C, lizuka T, et al. Treatment and prognostic factors for long-term outcome in patients with anti-NMDA receptor encephalitis: an observational cohort study. Lancet Neurol 2013; 12: 157-65.

4 Herken J, Pruss $\mathrm{H}$. Red flags: clinical signs for identifying autoimmune encephalitis in psychiatric patients. Front Psychiatry 2017; 8: 25.

5 Byrne S, Walsh C, Hacohen Y, Muscal E, Jankovic J, Stocco A, et al. Earlier treatment of NMDAR antibody encephalitis in children results in a better outcome. Neurol Neuroimmunol Neuroinflammation 2015; 2: e130.

6 Espinola-Nadurille M, Flores-Rivera J, Rivas-Alonso V, Vargas-Cañas S, Fricchione $\mathrm{GL}$, Bayliss $\mathrm{L}$, et al. Catatonia in patients with anti-NMDA receptor encephalitis. Psychiatry Clin Neurosci 2019; 73: 574-80.

7 Rogers JP, Pollak TA, Blackman G, David AS. Catatonia and the immune system: a review. Lancet Psychiatry 2019; 6: 620-30.

8 Bush G, Fink M, Petrides G, Dowling F, Francis A. Catatonia. I. Rating scale and standardized examination. Acta Psychiatr Scand 1996; 93: 129-36.

9 Walther S, Stegmayer K, Wilson JE, Heckers S. Structure and neural mechanisms of catatonia. Lancet Psychiatry 2019; 6: 610-9.

10 Riss J, Cloyd J, Gates J, Collins S. Benzodiazepines in epilepsy: pharmacology and pharmacokinetics. Acta Neurol Scand 2008; 118: 69-86.

11 Dalmau J, Lancaster E, Martinez-Hernandez E, Rosenfeld MR, Balice-Gordon R Clinical experience and laboratory investigations in patients with anti-NMDAR encephalitis. Lancet Neurology 2011; 10: 63-74.

12 Mohammad SS, Jones $H$, Hong $M$, Nosadini $M$, Sharpe $C$, Pillai SC, et al. Symptomatic treatment of children with anti-NMDAR encephalitis. Dev Med Child Neurol 2016; 58: 376-84

13 DeSena AD, Greenberg BM, Graves D. Three phenotypes of anti-N-methyl-Daspartate receptor antibody encephalitis in children: prevalence of symptoms and prognosis. Pediatr Neurol 2014; 51: 542-9. 